\title{
On the Distributions of Subgraph Centralities in Complex Networks
}

\author{
Faxu Li, ${ }^{1,2}$ Liang Wei, ${ }^{3}$ Haixing Zhao, ${ }^{2}$ and Feng $\mathrm{Hu}^{2}$ \\ ${ }^{1}$ School of Computer Science, Shaanxi Normal University, Xian, Shanxi 710062, China \\ ${ }^{2}$ College of Computer, Qinghai Normal University, Xining, Qinghai 810008, China \\ ${ }^{3}$ Department of Mathematics, Qinghai Normal University, Xining, Qinghai 810008, China
}

Correspondence should be addressed to Haixing Zhao; h.x.zhao@163.com

Received 15 August 2013; Accepted 12 September 2013

Academic Editor: Jinde Cao

Copyright (C) 2013 Faxu Li et al. This is an open access article distributed under the Creative Commons Attribution License, which permits unrestricted use, distribution, and reproduction in any medium, provided the original work is properly cited.

\begin{abstract}
Subgraph centrality measure characterizes the participation of each node in all subgraphs in a network. Smaller subgraphs are given more weight than large ones, which makes this measure appropriate for characterizing network motifs. This measure is better in being able to discriminate the nodes of a network than alternate measures. In this paper, the important issue of subgraph centrality distributions is investigated through theory-guided extensive numerical simulations, for three typical complex network models, namely, the ER random-graph networks, WS small-world networks, and BA scale-free networks. It is found that these three very different types of complex networks share some common features, particularly that the subgraph centrality distributions in increasing order are all insensitive to the network connectivity characteristics, and also found that the probability distributions of subgraph centrality of the ER and of the WS models both follow the gamma distribution, and the BA scale-free networks exhibit a power-law distribution with an exponential cutoff.
\end{abstract}

\section{Introduction}

Complex networks, consisting of sets of nodes or vertices joined together in pairs by links or edges, appear frequently in various technological, social, and biological scenarios [1-5]. These networks include the Internet [6], the World Wide Web [7], social networks [8-10], scientific collaboration networks [11], lexicon or semantic networks [12, 13] and neural networks [14], food webs [15], metabolic networks [16], and protein interaction networks [17]. They have been shown to share global statistical features, such as the "small world" and the "scale-free" effects, as well as the "clustering" property.

It has been observed that not only triangles but also other subgraphs are significant in real networks. We say that a graph $G^{\prime}=\left(V^{\prime}, E^{\prime}\right)$ is a subgraph of $G=(V, E)$ if $V^{\prime} \subseteq V$ and $E^{\prime} \subseteq E$. The term "network motifs" designates those patterns that occur in the network far more often than in random networks with degree sequence [18]. Network motifs found in technological and biological networks are small subgraphs that capture specific patterns of interconnection characterizing the networks at the local level $[18,19]$.

Another kind of local characterization of networks is made numerically by using one of several measures known as "centrality" [20]. One of the most used centrality measures is the "degree centrality", DC [7], which is a fundamental quantity describing the topology of scale-free networks [21].

There are several other centrality measures that have been introduced and studied for real-world networks, such as closeness, betweenness, eigenvector and subgraph centrality, and so on. They account for the different node characteristics that permit them to be ranked in the order of importance in the network. Among these centrality measures, subgraph centrality characterizes nodes according to their participation in structural subgraphs in the network, giving higher weights to the smaller subgraphs that can be involved in network motifs. This measure has been tested in artificial networks, 
showing that it is better in being able to discriminate the nodes of a network than alternate measures [22].

Among the many representative network models, the classical Erdös-Rényi (ER) random-graph networks [23], Watts-Strogatz (WS) small-world networks [14] and Barabási-Albert (BA) scale-free networks [24] are particularly significant and important. The questions to be addressed in this paper are as folloiws: "are there any similar statistical properties of the subgraph centralities with respect to the topological features in ER, WS, and BA network models? What probability distributions do the subgraph centrality of the three network models follow?" Noticeably, the two questions have been addressed from different points of view in the literature. For example, it was shown in [22] that the cumulative distribution of subgraph centrality displayed scale-free characteristics in the eight networks studied. From a somewhat different standpoint and approach, this paper performs a careful numerical analysis based on intensive computer simulations to study the subgraph centrality distributions of three representative complex network models: the ER random-graph networks, WS small-world networks, and BA scale-free networks.

One of the main findings of this paper is that the probability distributions of subgraph centrality for the ER and WS models both follow the gamma distribution, and the BA scale-free networks exhibit a power-law distribution with an exponential cutoff; another finding is that the subgraph centrality distributions in increasing order of the aforementioned three types of complex networks have very different properties in general and yet meanwhile share some common features. More precisely, by plotting the subgraph centrality indices in increasing order of three network models, we obtain various "subgraph centrality curves" and find that, in different realizations of the same type of network topology of the same size, the relative deviations of their subgraph centrality curves are very small with only up to \pm 0.5 errors. This phenomenon shows that, for a certain network with fixed size and given node-degree distribution, the subgraph centrality distributions of these three very different types of complex networks are all insensitive to the network connectivity characteristics.

The rest of this paper is organized as follows. In Section 2, we provide a brief definition of subgraph centrality and generating algorithms for the ER random-graph networks, WS small-world networks, and BA scale-free networks, useful throughout the paper. Section 3 presents some theory-guided numerical analysis of the subgraph centrality distributions of three network models in the nondecreasing order. Section 4 gives some theory-guided numerical analyses about the probability distributions of subgraph centrality for three network models. The main results of the paper reported in Sections 3 and 4. Finally, conclusions are drawn in Section 5.

\section{Preliminaries}

2.1. Notations. $N$ is the the number of nodes in a network model. $p$ is the probability parameter. $k_{i}$ is the degree of node $i$, defined to be the number of edges directly connecting node $i$. $A$ is the $N \times N$ adjacency matrix, defined by

$$
a_{i j}= \begin{cases}1 & \text { if } i \neq j, i \text { adjacent } j \\ 0 & \text { otherwise. }\end{cases}
$$

2.2. Subgraph Centrality $\left(C_{S}\right)$. Subgraph centrality characterizes the participation of each node in all subgraphs in a network. The subgraph centrality of node $i$ was defined as the "sum" of closed walks of different lengths in the network starting and ending at node $i$. The contribution of these closed walks decreases as the length of the walks increases. That is, shorter closed walks have more influence on the centrality of the node than longer closed walk. The subgraph centrality of node $i$ in the networks is given by [22]

$$
C_{S}(i)=\sum_{k=0}^{\infty} \frac{\mu_{k}(i)}{k !} .
$$

By a series of deducing, the subgraph centrality for all $i \in V$ may be expressed as follows [22]:

$$
C_{S}(i)=\sum_{j=1}^{N} v_{j}^{i} e^{\lambda_{j}},
$$

where $v_{1}, v_{2}, \ldots, v_{N}$ are an orthonormal basis of $R^{N}$ composed by eigenvectors of $A$ associated with the eigenvalues $\lambda_{1}, \lambda_{2}, \ldots, \lambda_{N}$, and $v_{j}^{i}$ denotes the $i$ th component of $v_{j}$.

A global characterization of the network can be carried out by means of the average subgraph centrality, denoted by $\left\langle C_{S}\right\rangle$. It is given by [22]

$$
\left\langle C_{S}\right\rangle=\frac{1}{N} \sum_{i=1}^{N} C_{S}(i)=\frac{1}{N} \sum_{i=1}^{N} e^{\lambda_{i}} .
$$

It was shown that $\left\langle C_{S}\right\rangle$ depends only on the eigenvalues and size of the adjacency matrix of the network [22].

\subsection{Generation Algorithms of Complex Network Models}

2.3.1. The ER Random-Graph Network. The ER randomgraph network is generated by the following steps (ER algorithm); see [25].

(1) Start with $N$ isolated nodes, and number the nodes from 0 to $(N-1)$.

(2) Pick up every pair of nodes and connect them by edge with probability $p$.

2.3.2. The WS Small-World Network. The WS small-world network model is generated by the following steps (WS algorithm); see [25].

(1) Start with a regular nearest-neighboring network with $N$ nodes, in which each node is connected to its $2 m_{0}$ neighbors, where $m_{0}>0$ is a small integer. 
(2) At every step, for each node, operate on its connections to its $m_{0}$ nearest neighbors, one by one in a clockwise (or counterclockwise) manner: an edge connecting this node to its neighbor will be kept in place, but another end of edge will be reconnected with probability $p$ to a node randomly chosen from elsewhere in the network.

2.3.3. The BA Scale-Free Network Model. The BA scale-free network model is generated by the following steps (BA algorithm); see [25].

(1) Growth: starting from a small fully connected network of nodes $m_{0}>0$, where $m_{0}>0$ is a small integer. At each time step, a new node is added to the existing network, and this new node is connected to $m$, the previously added nodes, where $1 \leq m \leq m_{0}$.

(2) Preferential attachment: the probability of a new link to end up in an existing node $i$ is proportional to the connectivity $k_{i}$ of this node:

$$
\prod\left(k_{i}\right)=\frac{k_{i}}{\sum_{j=1}^{N} k_{j}} .
$$

\section{Distributions of Subgraph Centralities in the Increasing Order}

In this section, we discuss the distributions of subgraph centralities calculated by (3) for three typical network models, namely, the ER random-graph model, the WS small-world model and the BA scale-free model, respectively. To quantify the variability of a subgraph centrality, define the relative deviation as

$$
v_{i}=\frac{C_{S}(i)-\left\langle C_{S}(i)\right\rangle}{\left\langle C_{S}(i)\right\rangle},
$$

where $\left\langle C_{S}(i)\right\rangle$ is averaged over $C_{S}(i)$ of 50 networks, and $\left\langle C_{S}(i)\right\rangle \neq 0$ for all $i, i=1,2, \ldots, N$.

3.1. ER Random-Graph Networks. The simulation results are plotted in Figure 1(a), where the number of simulation runs on ER random-graph network model is 50, therefore totally 50 network realizations were generated by ER algorithm, and a total of $2500 \times 50$ subgraph centralities were plotted altogether in the same figure (they are not the averages of the subgraph centralities of the 50 simulations). Figure 1(b) shows the averages of the subgraph centralities of the ER model plotted in Figure 1(a). To quantify the variability of the subgraph centralities, the relative deviations defined by (6) were calculated and are plotted in Figure 2. The ER algorithm was run with $N=2500$ and $p=0.08$.

The simulation results reveal the following observations.

(1) It is clear, from a comparison of Figure 1(a) with Figure 1(b), that the two figures look quite similar; namely, the actual values of each subgraph centrality in all individual runs and averages of the subgraph

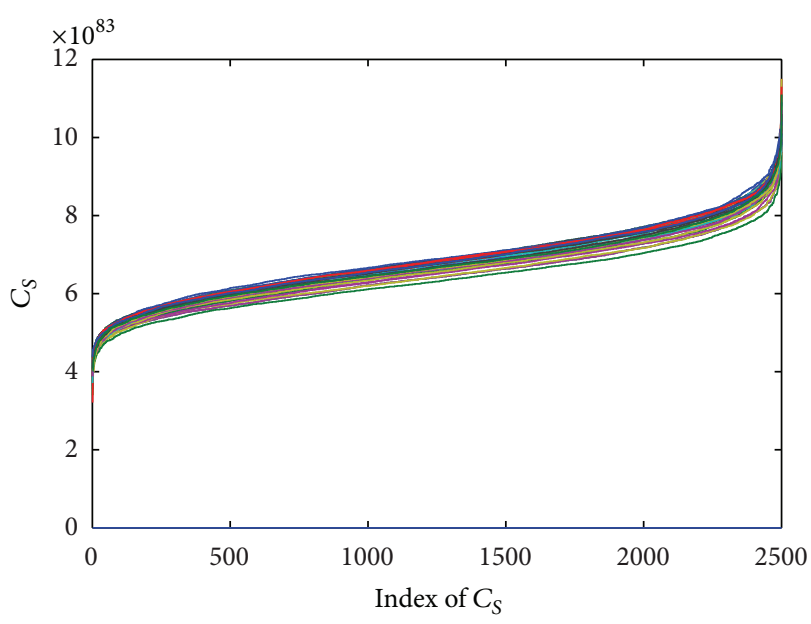

(a)

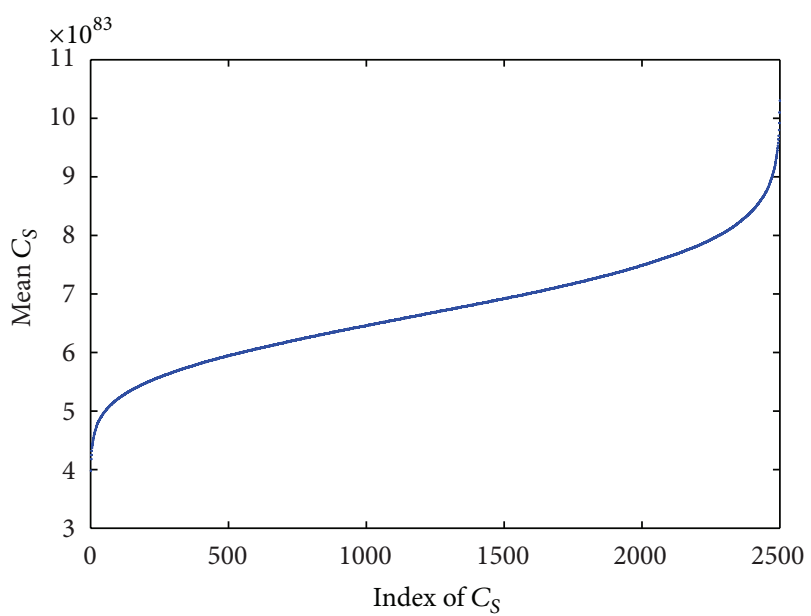

(b)

FIgURE 1: (a) The subgraph centrality $C_{S}(i)$ curves of the ER model, (b) mean subgraph centrality $\left\langle C_{S}(i)\right\rangle$ curve of the ER model.

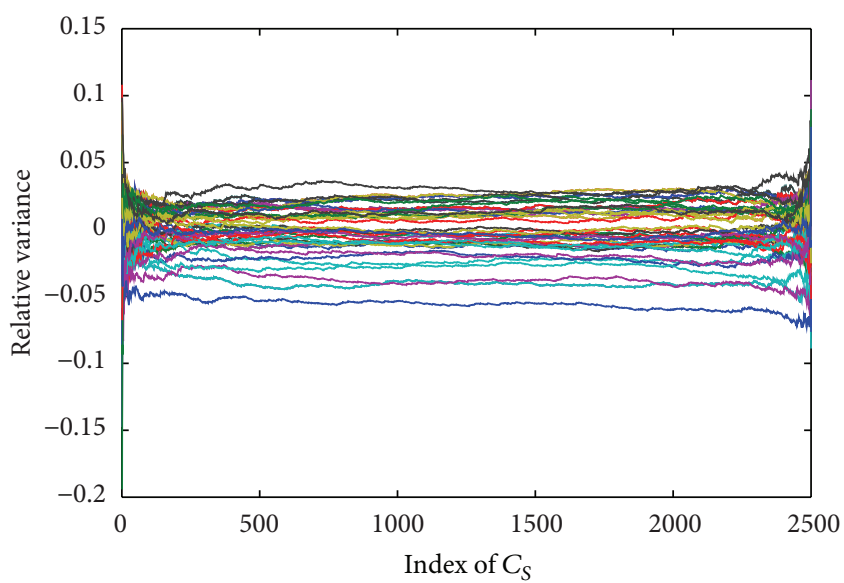

FIGURE 2: Relative deviations of subgraph centralities $C_{S}(i)$ of the ER model. 


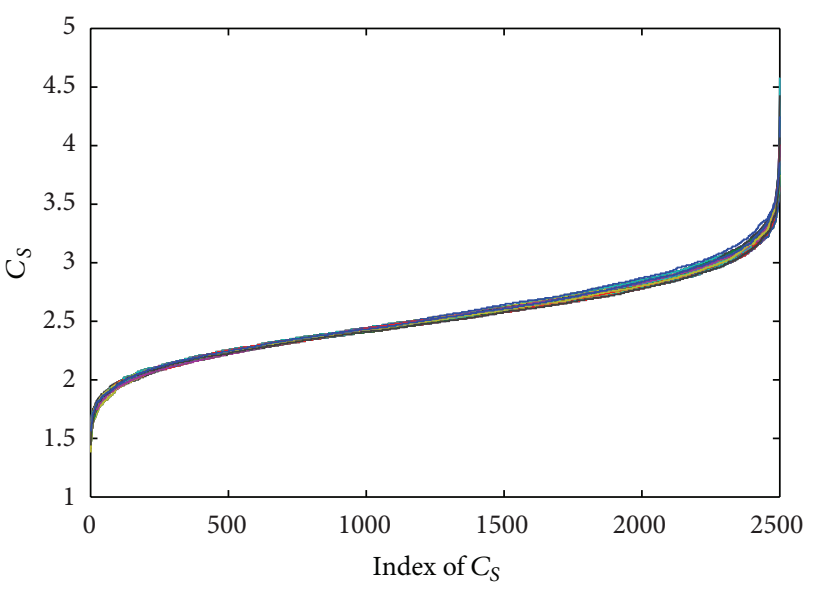

(a)

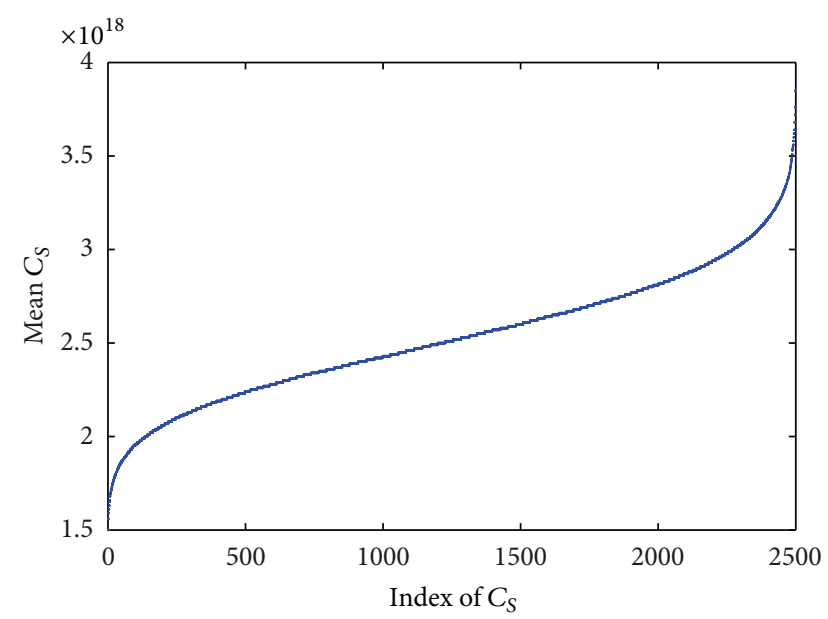

(b)

FIgURE 3: (a) The subgraph centrality $C_{S}(i)$ curves of the WS smallworld network, (b) mean subgraph centrality $\left\langle C_{S}(i)\right\rangle$ curve of the WS small-world network.

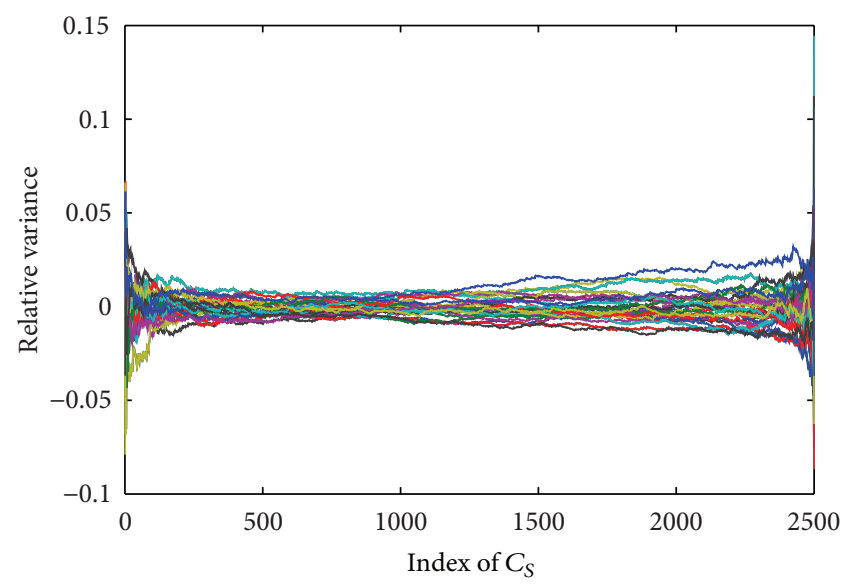

FIGURE 4: Relative deviations of subgraph centralities $C_{S}(i)$ of the WS small-world network model.

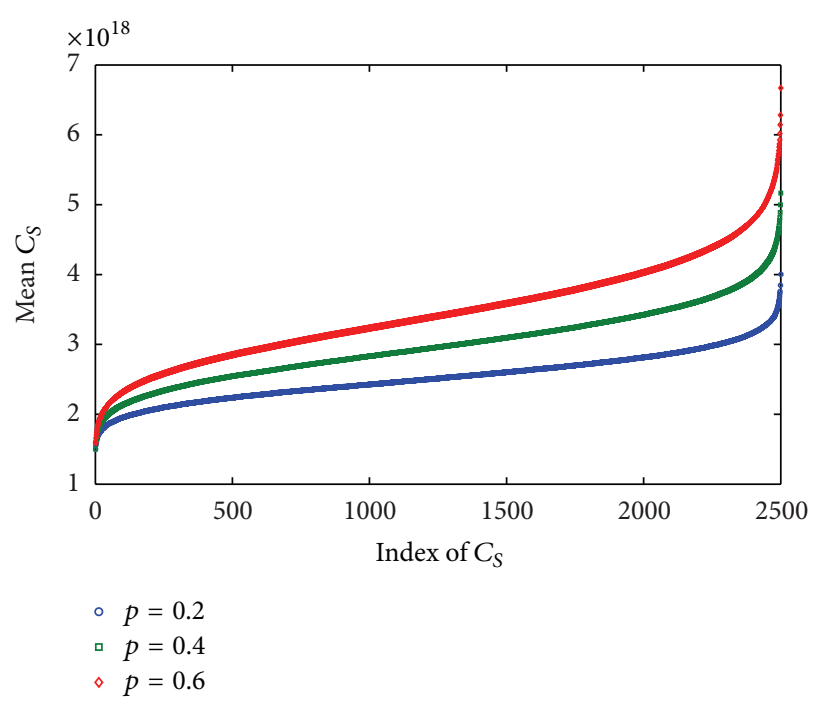

FIGURE 5: The mean subgraph centrality $\left\langle C_{S}(i)\right\rangle$ curves of the WS small-world network with three rewiring probabilities $p=0.2$ (red circles), $p=0.4$ (green squares), and $p=0.6$ (blue diamonds). All networks have $N=2500$. All data are averaged over 50 networks.

centralities are not much different. This implies that in various realizations of the same type of network topology of the same size, the variation of their subgraph centralities is quite small. A phenomenon needs further investigation in the future.

(2) Figure 2 shows that the relative deviations of subgraph centralities are mostly located nearby, with up to \pm 0.05 errors, which are quite small. This phenomenon shows that the subgraph centralities of ER complex networks are insensitive to the network connectivity characteristics; namely, in different realizations with the same size $N$ and probability $p$, the subgraph centrality curves are almost the same as shown in Figure 1. Thus, for an ER-type complex network, in order to estimate its subgraph centrality distribution, one does not need to know the detailed information about its specific connectivity topology but only needs to know the values of $N$ and $p$ in general.

3.2. WS Small-World Networks. Similar to the analysis of ER complex networks, Figure 3 shows the plots of the curves of the distributions of subgraph centralities and mean subgraph centralities. Figure 4 shows the plot of the relative deviations. The WS algorithm was run with $N=2500, m_{0}=25$ and $p=0.2$.

The simulation results reveal the following observations.

(1) It is clear, from a comparison of Figure 3(a) with Figure 3(b), that the two figures look quite similar. This implies that in various realizations of the same type of network topology of the same size, the variation of their subgraph centralities is quite small.

(2) Figure 4 shows that the relative deviations of subgraph centralities are mostly located nearly, with 


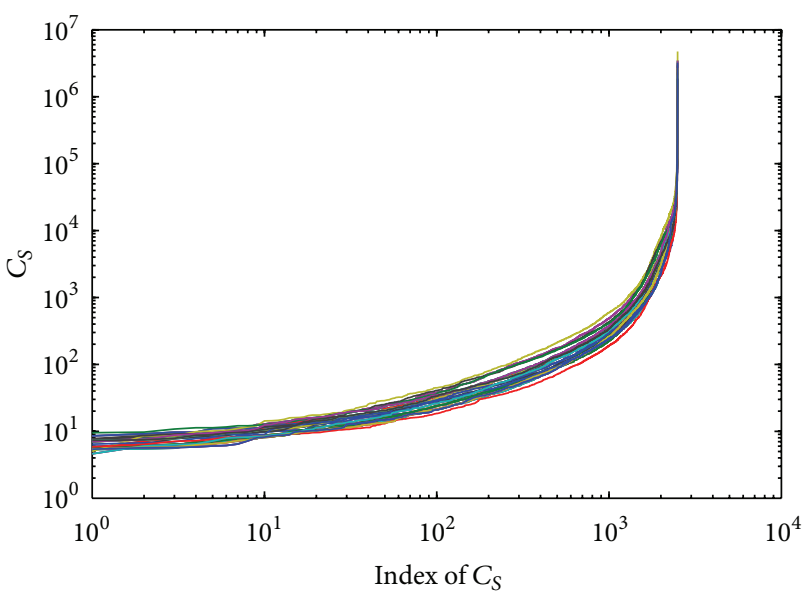

(a)

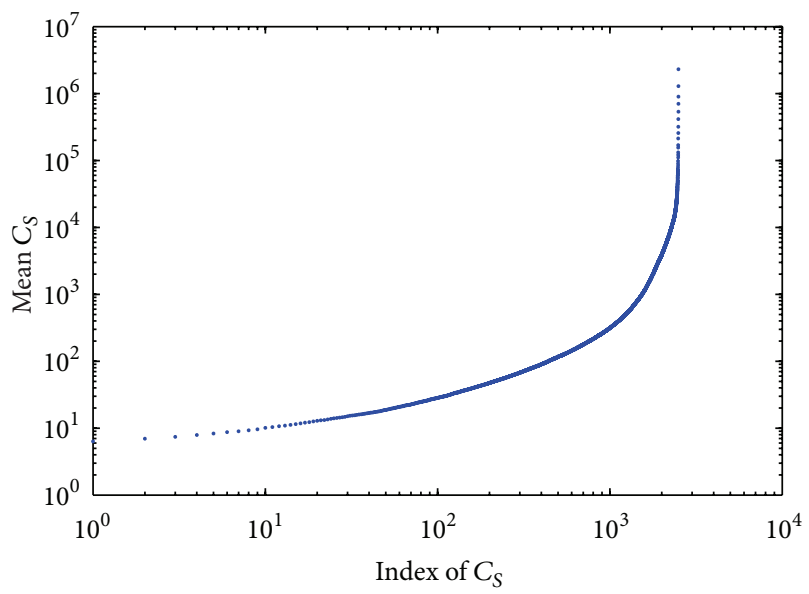

(b)

FIGURE 6: (a) Log-log plot of the subgraph centrality $C_{S}(i)$ in the BA scale-free network, (b) log-log plot of mean subgraph centrality $\left\langle C_{S}(i)\right\rangle$ in the BA scale-free network.

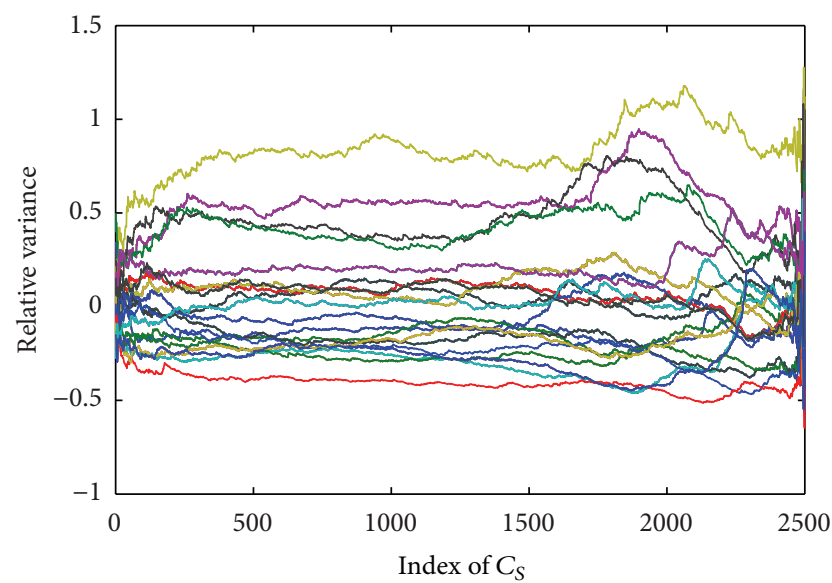

FIGURE 7: Relative deviations of subgraph centralities $C_{S}(i)$ of the BA scale-free network.

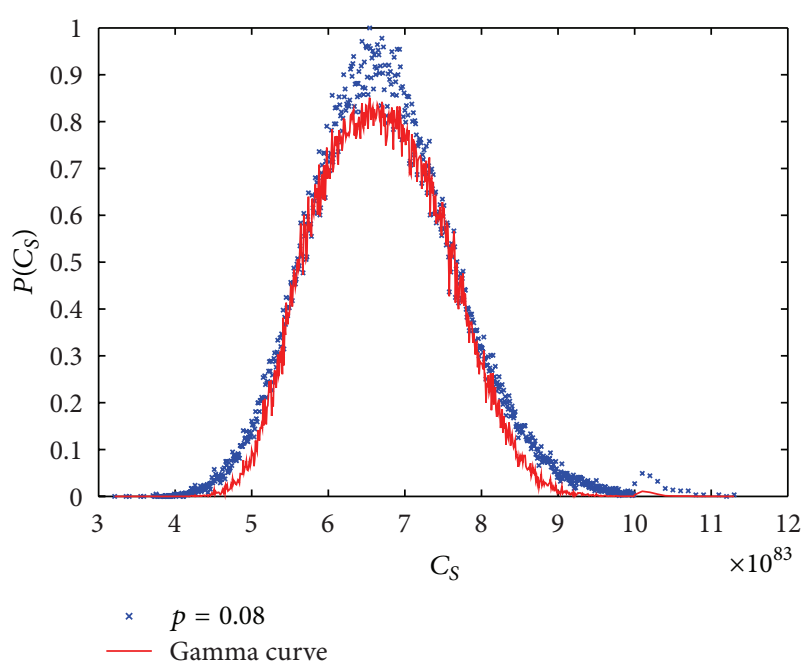

(a)

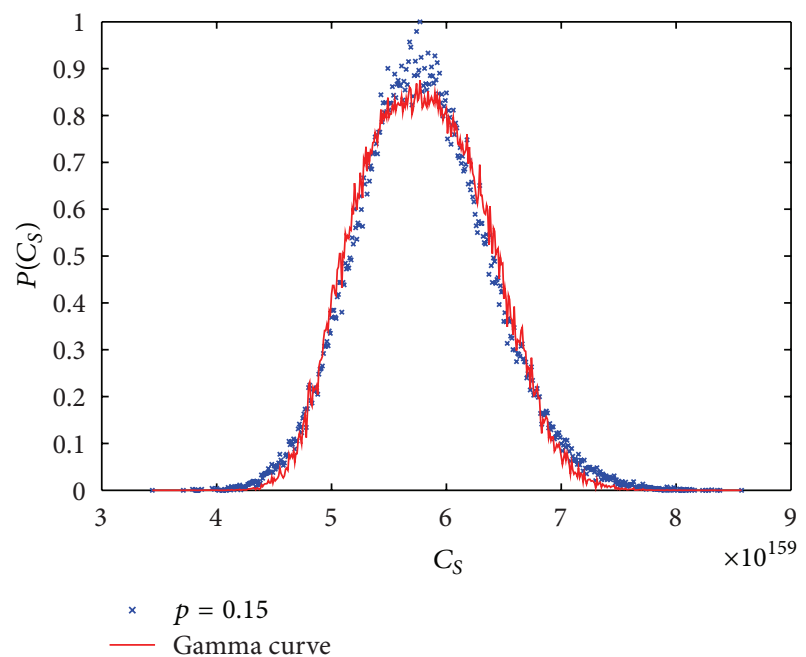

(b)

FIGURE 8: Probability distributions of subgraph centrality $P\left(C_{S}\right)$ and the fitting curve of gamma distribution for the ER network model. (a) $P\left(C_{S}\right)$ for $N=2500$ and $p=0.08$, gamma distribution with $\alpha=2.07$ and $\beta=0.28$. (b) $P\left(C_{S}\right)$ for $N=2500$ and $p=0.15$, gamma distribution with $\alpha=1.74$ and $\beta=0.31$. All networks have $N=2500$.

up to \pm 0.025 errors, which are quite small. This phenomenon shows that the subgraph centralities of WS complex networks are insensitivite to the network connectivity characteristics; namely, in different realizations with the same size $N$, parameter $m_{0}$, and rewiring probability $p$, the subgraph centrality curves are almost the same as shown in Figure 3. Thus, for an WS-type complex network, in order to estimate its subgraph centrality distribution, one does not need to know the detailed information about its specific connectivity topology but only needs to know the values of $N, m_{0}$, and $p$.

(3) If $N=2500$ and $m_{0}$ are both fixed but the rewiring probability $p$ is increased, then the mean subgraph 


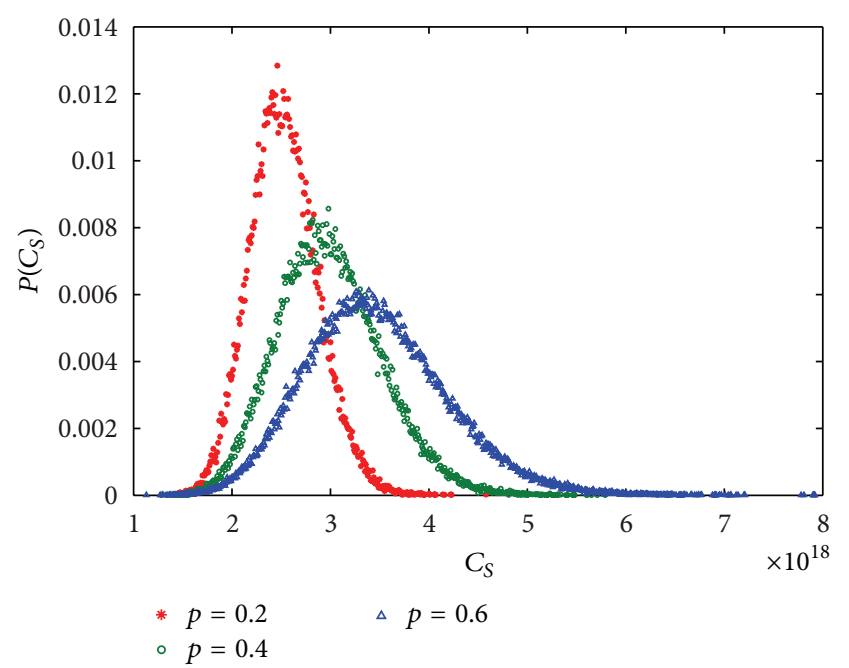

(a)

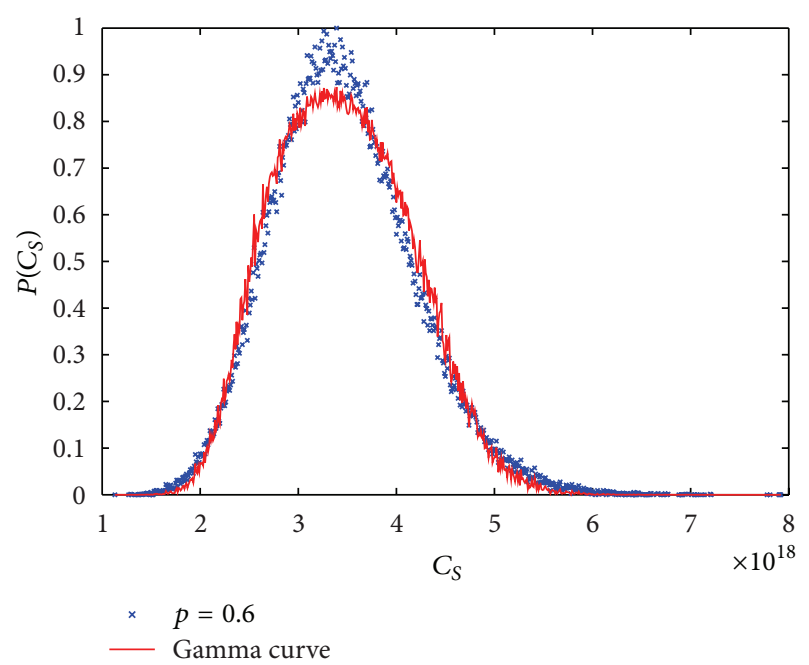

(b)

FIGURE 9: (a) Probability distributions of subgraph centrality $P\left(C_{S}\right)$ in the WS network model with three rewiring probabilities $p=0.2$ (red circles), $p=0.4$ (green squares), and $p=0.6$ (blue diamonds). (b) Probability distributions of subgraph centrality $P\left(C_{S}\right)$ with $p=$ 0.6 and the fitting curve of gamma distribution with $\alpha=1.62$ and $\beta=0.33$. All networks have $N=2500$.

centrality curve has some gradual change in shape. The $\left\langle C_{S}(i)\right\rangle$ increases as the rewiring probability $p$ increases, as shown in Figure 5. This phenomenon shows that the rewiring probability $p$ can be used to control the "regular" and "random" levels of the WS small-world network model, which is consistent with our understanding of the model [14]: when $p$ is small, which means only a few edges are rewired, the network generated by the WS algorithm is more like a regular network; but, as $p$ is increased, more edges will be rewired, so the generated network becomes more random; when $p=1$, every edge will be rewired; therefore, the WS small-world network model literally becomes an ER random-graph network. Thus, by

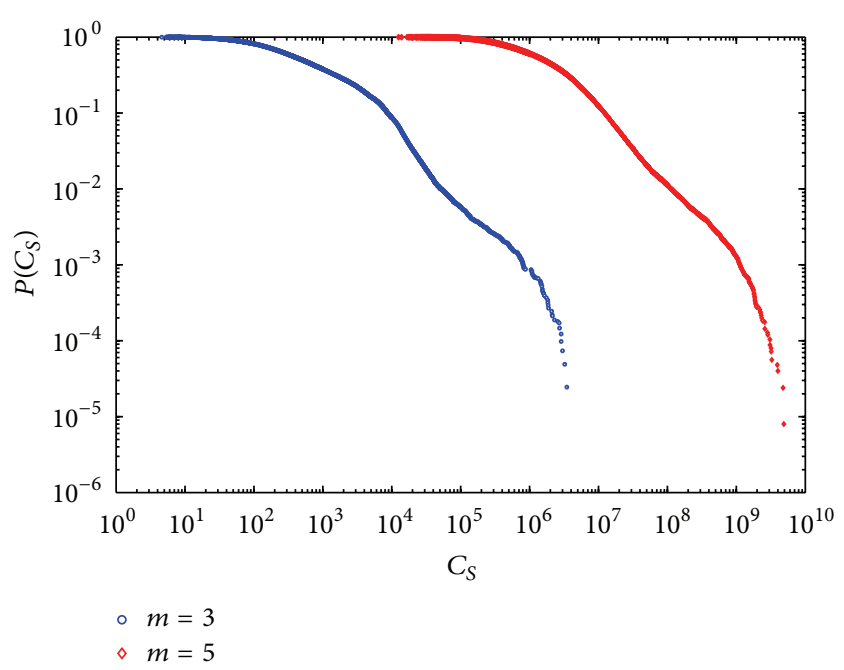

Figure 10: (Color online) Log-log plot of the cumulative distributions of $P\left(C_{S}\right)$ for the BA network model with $m=3$ (blue circles) and $m=5$ (red squares). All networks have $N=2500$.

tuning the value of $p$, one can obtain a transition from a completely regular network to a completely random network.

3.3. BA Scale-Free Networks. The curves of the distributions of subgraph centralities, mean subgraph centralities, and the relative deviations are plotted in Figures 6 and 7, respectively. It was shown [21] that scale-free characteristics of BA networks are independent of three parameters $N, m_{0}$, and $m$. So the algorithm of BA model was run with $N=2500, m_{0}=5$, and $m=3$.

The simulation results reveal the following observations.

(1) It is clear, from a comparison of Figure 6(a) with Figure 6(b), that the two figures look quite similar. This implies that, in various realizations of the same type of network topology of the same size, the variation of their subgraph centralities is quite small.

(2) Figure 7 shows that the relative deviations of subgraph centralities are mostly located nearly, with up to \pm 1 errors; I just found that the relative deviations of BA network are really much more sensitive than the other two kinds of networks. This phenomenon shows that the subgraph centralities of BA complex networks are insensitivite to the network connectivity characteristics; namely, in different realizations with the same size $N$, parameter $m_{0}$, and $m$, the subgraph centralities curves are almost the same.

(3) Figure 6 shows that there is a small number with high $C_{S}(i)$ nodes and a large number of low $C_{S}(i)$ nodes in network. This phenomenon shows the scale-free characteristics of BA networks. 


\section{Probability Distributions of Subgraph Centralities}

In this section, the probability distributions of subgraph centrality are discussed for the aforementioned three different types of networks.

4.1. ER Random-Graph Networks. The simulation results are averaged over 50 network realizations. In Figure 8, we plot the probability distributions of subgraph centrality of the ER random-graph model for different parameters.

The simulation results show that the plots of $P\left(C_{S}\right)$ are quite similar under different parameters $p$. We use $P$ - $P$ plots in IBM SPSS Statistics 20.0 to test these simulation data. The distributions like gamma distribution, it also looks like normal distribution, or $t$-distribution. We find that bias of the gamma distribution is minimum almost three distributions, so we conclude that the distributions of subgraph centrality follow the gamma distribution. Probability distributions of subgraph centrality $P\left(C_{S}\right)$ from these simulation data perfectly fit the gamma distribution, where $P\left(C_{S}\right)$ values are normalized; see Figure 8.

4.2. WS Small-World Networks. Similar to the simulation of ER complex networks, Figure 9 shows the plot of the distributions of subgraph centrality under different parameters $p$.

The simulation results reveal the following observations.

(1) Figure 9(a) shows that the curves of $P\left(C_{S}\right)$ are very similar under different parameters $p$ in general, and yet meanwhile they also display different features. The distribution of subgraph centrality flattens and widens as the rewiring probability $p$ increases. This phenomenon shows that the rewiring probability $p$ can be used to control the "regular" and "random" levels of the WS small-world network model: when the rewiring probability $p$ is small, which means only a few edges are rewired, the network generated by the WS algorithm is more like a regular network; but, as $p$ is increased, more edges will be rewired, so the generated network becomes more random; when $p=1$, every edge will be rewired therefore the WS small-world network model literally becomes an ER random-graph network. This phenomenon shows that small worlds are hybrids-part regular and part random. The topology of a small-world network should fall somewhere between that of regular network and random network. A small-world network is taller and thinner than the distribution of equivalent random network.

(2) From simulation results, we conclude that the distributions of subgraph centrality follow the gamma distribution. Probability distributions of subgraph centrality $P\left(C_{S}\right)$ from these simulation data perfectly fit the gamma distribution, where $P\left(C_{S}\right)$ values are normalized; see Figure 9(b).

4.3. BA Scale-Free Networks. The simulation results are averaged over 50 network realizations. In Figure 10, we plot the cumulative distributions of subgraph centrality for different parameters $m$.

From simulations, it is found that the cumulative distributions of $P\left(C_{S}\right)$ display a power-law distribution with an exponential cutoff. The scale-free characteristics of $C_{S}(i)$ distribution can be explained as follows. $C_{S}(i)$ measures the number of times that a node participates in all subgraphs in the network, giving more weight to smaller subgraphs. Consequently, nodes with high $C_{S}(i)$ participate in large number of small subgraphs, such as connected triplets, triangles, squares, and so forth. The frequency of these nodes in the network is significantly higher than that of nodes participating in a small number of subgraphs or participating only in large subgraphs from which a fat tail distribution results.

\section{Conclusion}

In this paper, both subgraph centrality distributions in the increasing order and probability distributions of subgraph centrality for three representative complex network models have been carefully investigated through extensive theoryguided simulations, including the ER random-graph networks, WS small-world networks, and BA scale-free networks. The results show that these three very different types of complex networks share some common features; particularly, similarities in the subgraph centrality distributions in the increasing order are all insensitive to the network connectivity characteristics. It also shows that the probability distributions of subgraph centrality of the ER and of the WS models both follow the gamma distribution, and the BA scale-free networks exhibit a power-law distribution with an exponential cutoff.

\section{Conflicts of Interests}

The authors declare that there is no conflict of interests regarding the publication of this paper.

\section{Acknowledgments}

The research is supported by the National Science Foundation of China (nos. 61164005, 60863006), the National Basic Research Program of China (no. 2010CB334708), and the Program for Changjiang Scholars and Innovative Research Team in University (no. IRT1068).

\section{References}

[1] E. Estrada and J. A. Rodríguez-Velázquez, "Subgraph centrality in complex networks," Physical Review E, vol. 71, no. 5, Article ID 056103, 9 pages, 2005.

[2] S. H. Strogatz, "Exploring complex networks," Nature, vol. 410, no. 6825 , pp. $268-276,2001$.

[3] P. F. M. Faloutsos and C. Faloutsos, "On power-law relationships of the Internet topology," Computer Communication Review, vol. 29 , no. 4, pp. 251-262, 1999. 
[4] R. Milo, S. Shen-Orr, S. Itzkovitz, N. Kashtan, D. Chklovskii, and U. Alon, "Network motifs: simple building blocks of complex networks," Science, vol. 298, no. 5594, pp. 824-827, 2002.

[5] M. E. J. Newman, "The structure and function of complex networks," SIAM Review, vol. 45, no. 2, pp. 167-256, 2003.

[6] R. J. Watts and N. D. Martinez, "Simple rules yield complex food webs," Nature, vol. 404, pp. 180-183, 2000.

[7] L. C. Freeman, "Centrality in social networks conceptual clarification,” Social Networks, vol. 1, no. 3, pp. 215-239, 1979.

[8] P. Erdős and A. Rényi, "On the evolution of random graphs," Mathematical Institute of the Hungarian Academy of Sciences, vol. 5, pp. 17-61, 1960.

[9] R. F. i Cancho and R. V. Solé, "The small world of human language," Proceedings of the Royal Society of London B, vol. 268, no. 1482, pp. 2261-2265, 2001.

[10] S. N. Dorogovtsev and J. F. F. Mendes, "Evolution of networks," Advances in Physics, vol. 51, no. 4, pp. 1079-1187, 2002.

[11] S. Wuchty, "Scale-free behavior in protein domain networks," Molecular Biology and Evolution, vol. 18, no. 9, pp. 1694-1702, 2001.

[12] D. J. Watts and S. H. Strogatz, "Collective dynamics of 'smallworld' networks," Nature, vol. 393, no. 6684, pp. 440-442, 1998.

[13] H. Jeong, B. Tombor, Z. N. Oltvai, and A.-L. Barabási, "The large-scale organization of metabolic networks," Nature, vol. 407, pp. 651-654, 2000.

[14] R. Albert, H. Jeong, and A.-L. Barabási, "Diameter of the worldwide web," Nature, vol. 401, no. 6749, pp. 130-131, 1999.

[15] S. Wasserman and K. Faust, Social Network Analysis, Cambridge University Press, Cambridge, UK, 1994.

[16] G. A. C. M. Sigman and G. A. Cecchi, "Global organization of the Wordnet lexicon," Proceedings of the National Academy of Sciences of the United States of America, vol. 99, no. 3, pp. 1742$1747,2002$.

[17] C. Zhan, G. Chen, and L. F. Yeung, "On the distributions of Laplacian eigenvalues versus node degrees in complex networks," Physica A, vol. 389, no. 8, pp. 1779-1788, 2010.

[18] D. J. Watts, Small Worlds: The Dynamics of Networks between Order and Randomness, Princeton Studies in Complexity, Princeton University Press, Princeton, NJ, USA, 1999.

[19] A. Schneeberger, C. H. Mercer, G. P. Garnett et al., "Scale-free networks and sexually transmitted diseases: a description of observed patterns of sexual contacts in Britain and Zimbabwe," Sexually Transmitted Diseases, vol. 31, no. 6, pp. 380-387, 2004.

[20] M. E. J. Newman, "The structure of scientific collaboration networks," Proceedings of the National Academy of Sciences of the United States of America, vol. 98, no. 2, pp. 404-409, 2001.

[21] A.-L. Barabási and R. Albert, "Emergence of scaling in random networks," Science, vol. 286, no. 5439, pp. 509-512, 1999.

[22] F. Liljeros, C. R. Edling, L. A. N. Amaral, H. E. Stanley, and Y. Åberg, "Social networks: the web of human sexual contacts," Nature, vol. 411, no. 6840, pp. 907-908, 2001.

[23] E. Yeger-Lotem, S. Sattath, N. Kashtan et al., "Network motifs in integrated cellular networks of transcription-regulation and protein-protein interaction," Proceedings of the National Academy of Sciences of the United States of America, vol. 101, pp. 5934-5939, 2004.

[24] R. Albert and A.-L. Barabási, "Statistical mechanics of complex networks," Reviews of Modern Physics, vol. 74, no. 1, pp. 47-97, 2002.
[25] A.-L. Barabási and Z. N. Oltvai, "Network biology: understanding the cell's functional organization," Nature Reviews Genetics, vol. 5, pp. 101-113, 2004. 


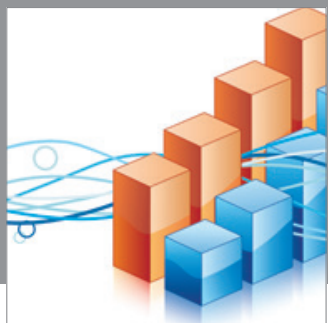

Advances in

Operations Research

mansans

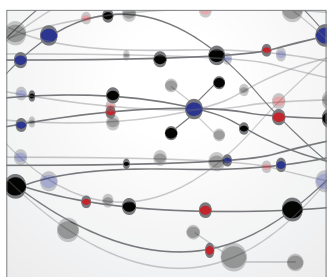

The Scientific World Journal
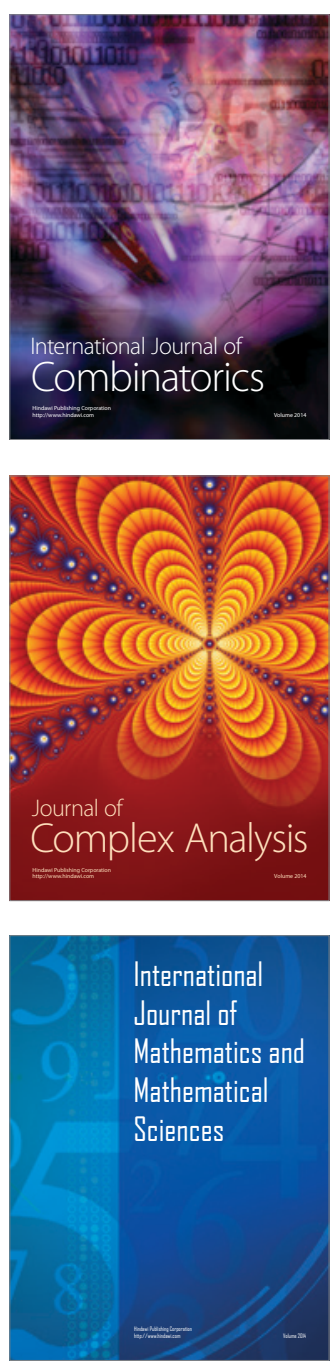
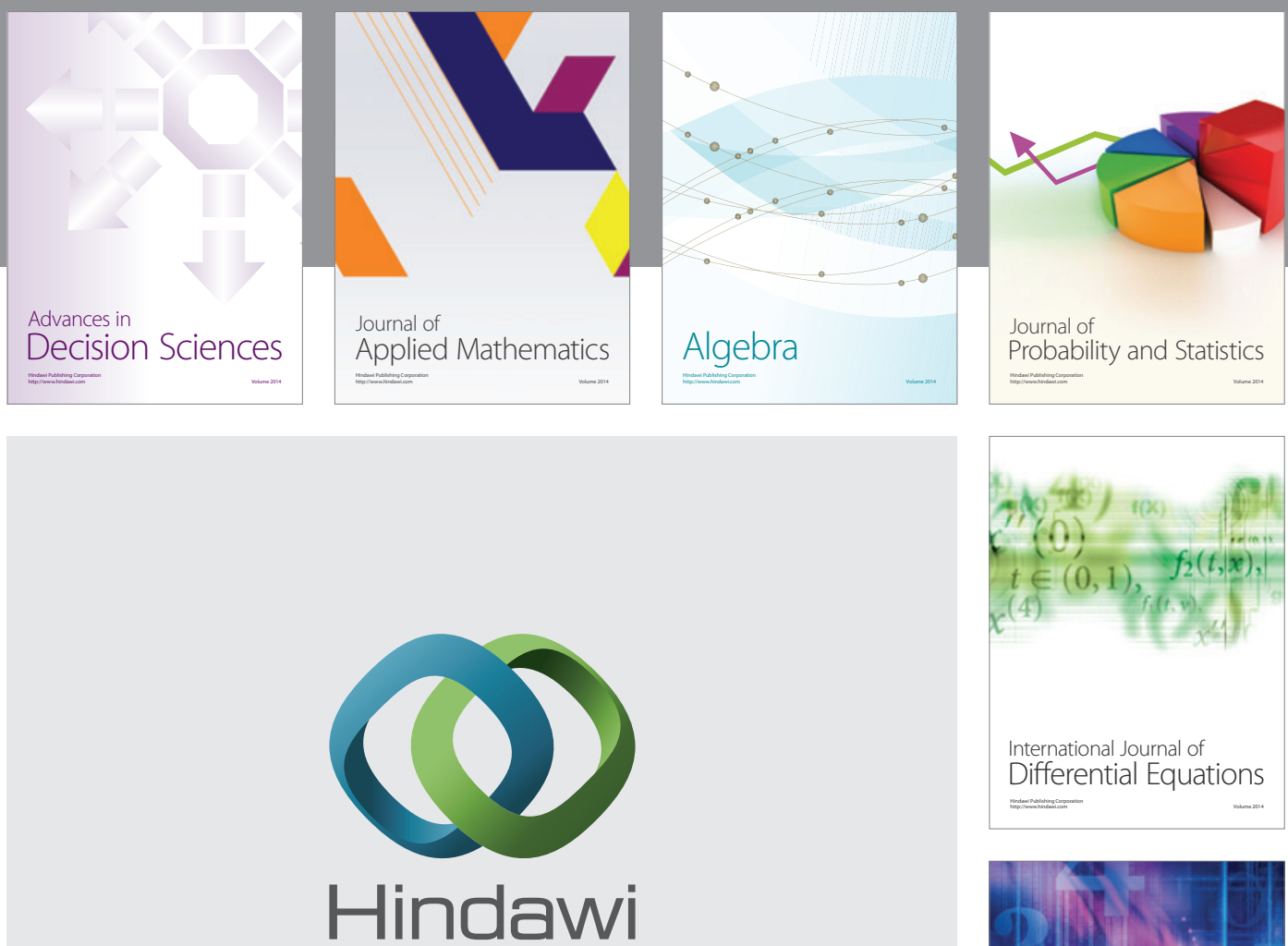

Submit your manuscripts at http://www.hindawi.com
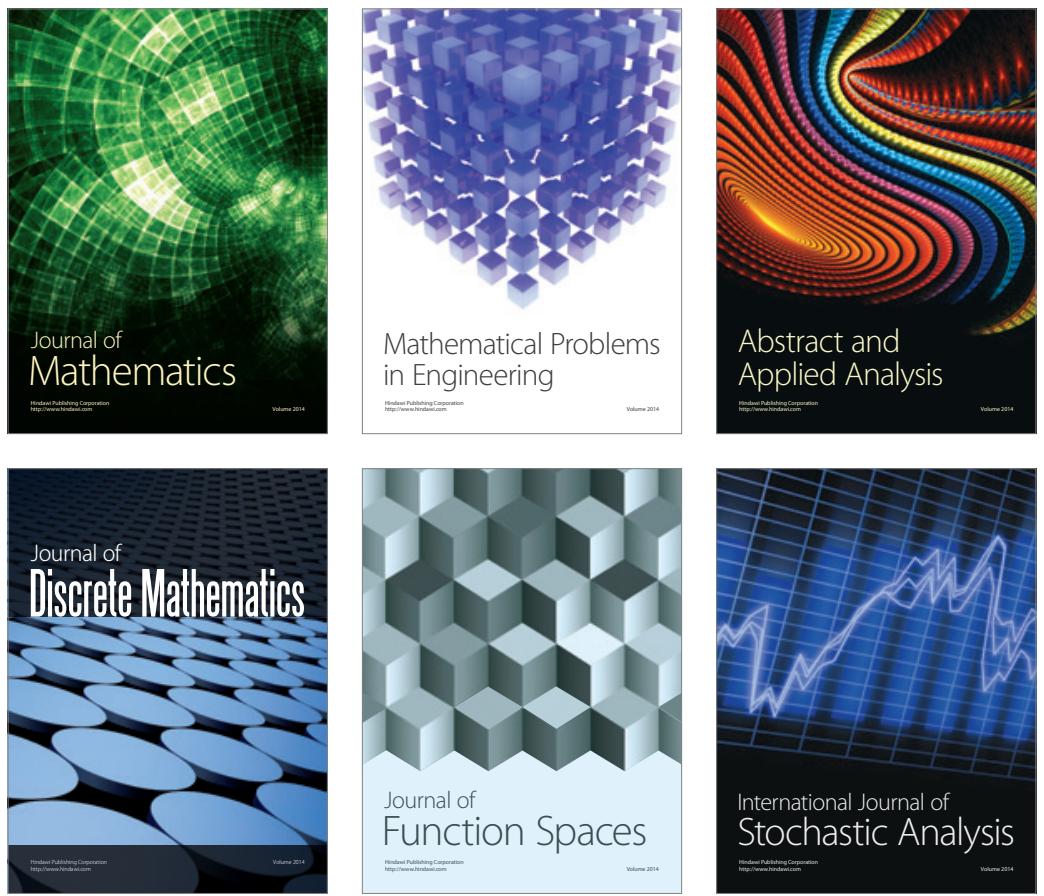

Journal of

Function Spaces

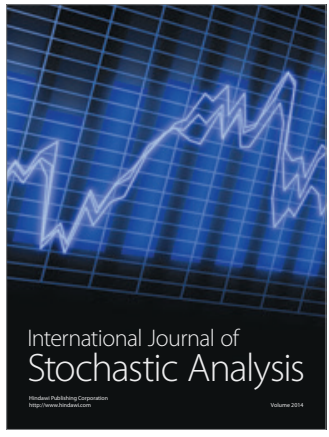

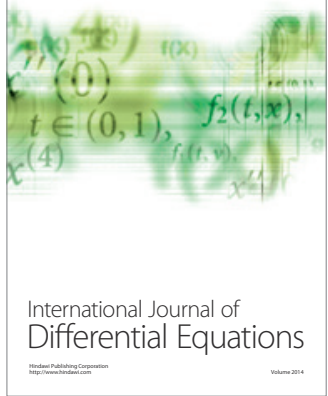
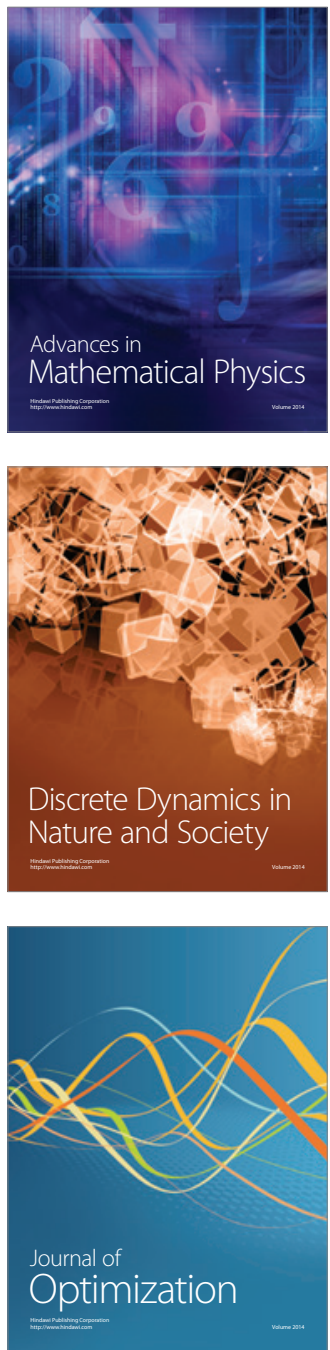